\title{
I-12. Spinal Arachnoid Diverticula
}

\author{
Katsumi Suematsu, Norio Mikami and Yuji Miyazaki \\ Department of Neurological Surgery, Sapporo Medical College and Hospital
}

The spinal arachnoid diverticula is intradural cyst in the dorsal aspect of spinal cord and has communication with the spinal subarachnoid space. The spinal arachnoid diverticula is able to encounter in myelogram sometimes the case of shown its clinical symptoms and signs is rare and only 40 cases of such spinal arachnoid diverticula have been reported in the literatures.

This congenital anomaly have to differentiate from a spinal cord tumor because of their clinical symptoms and signs. Spinal arachnoid diverticula can be differentiated from the spinal cord tumor by the remitting course of clinical symptom and myelographic findings. The myelogram of the spinal arachnoid diverticula shows typical pocket like contrast media retention in the supine or erect position and this evidence disappear in the prone or Trendelburg position.

The etiology of the spinal arachnoid diverticula is obscure and synomym have been used in the literatures, such as interadural cyst of the spinal meninges, leptmeningeal cyst, meningitis serosa circumscript spinalis etc.

Authors reported one case of 11 years old girl who has multiple spinal archnoid diverticula with remitting appearance and disappearance of weakness of both lower limbs.

\section{I-13. The Consideration on the Two Cases of Lumbar Lipomeningocele and the Fatty Tissue Connecting the Spinal Cord}

\author{
Shigeyuki Takaki, Shinken Kuramoto and Toru Shirouzu \\ Department of Neurosurgery, Kurume University, School of Medicine
}

The case of the Spina befida associated with the overgrowth of fatty tissue is recognized by many doctors, and it is included generally into the Spina bifida Occulta.

We had the two cases of Spina bifida accompanying the overgrowth of fatty tissue, operated them and investigated them morphologically. In this from we could confirm the existence of Spina bifida and the prolax of the meningeal sac with the fatty tissue from the spinal canal. Considering on it, they can't be formed only the lipoma in the spinal canal, but also the Spina bifida Occulta. By more investigation we came to consider it as lipomeningocele. 
In 1943 Ingraham and his comrades mentioned that the words of lipomeningocele or lipomeningomyelocele had been used. And in 1969 Emery and his comrades examined the lipoma of cauda equina and fatty tumor connecting the Spina bifida and they found the six forms.

Now we mention that there exist the forms are said as lipomeningocele or lipomeningomyelocele in the form which is called Spina bifida Occulta. Moreover we report the surgical treatment of this case and discuss about the fatty tissue associated with the spinal cord on the medical literature. 